\title{
Fine Tuning of a Wet Clutch Engagement by Means of a Genetic Algorithm
}

\author{
Yu Zhong ${ }^{1}$, Abhishek Dutta ${ }^{1}$, Bart Wyns $^{1}$, Clara-Mihaela Ionescu ${ }^{1}$, Gregory Pinte ${ }^{2}$, \\ Wim Symens ${ }^{2}$, Julian Stoev ${ }^{2}$, and Robin De Keyser ${ }^{1}$ \\ ${ }^{1}$ Department of Electrical energy, Systems and Automation (EeSA), \\ Ghent University, 913 Technologiepark, Zwijnaarde, 9052, Belgium \\ \{Yu.Zhong, Dutta.Abhishek, Bart.Wyns, \\ Claramihaela. Ionescu, Robain. Dekeyser\} @ugent. be \\ ${ }^{2}$ Flanders' Mechatronics Technology Center (FMTC), \\ Celestijnenlaan 300D, Heverlee, 3001, Belgium \\ \{Gregory.Pinte,Wim. Symens, Julian.Stoev\}@fmtc.be
}

\begin{abstract}
In many practical engineering applications, a feed-forward control is often used to control the system with some parameterized signals, for example, a wet clutch system. Usually these signals are designed empirically. In this paper, firstly, genetic algorithm (GA) will be used to optimize parameters. Then by knowing the system response of the test bench in the frequency domain, GA will be used again to fine tuning this parameterized signal. The result is then compared to those performances of using signal without fine tuning step. It is shown that after applying the fine tuning method, the resulted signal can achieve a better performance.
\end{abstract}

Keywords: Genetic algorithm, parameterized signal, discrete Fourier transform, system identification, wet clutch.

\section{$1 \quad$ Introduction}

A clutch is a mechanical device which provides the transmission of power/torque from one input component to an output component. This is a typical nonlinear system which also is very hard to model since inside the clutch, there are hydraulic, electronic and mechanical parts. Now the control strategy is feeding forward a parametric signal in which the parameters' values are set empirically. Usually with this method, the engagement performance is not optimal. In this paper, as a model free optimizing method, genetic algorithm (GA) will be implemented to tune the signal. After the tuning process, we can achieve a fast smooth engagement.

The paper is organized as follows: section 2 will introduce the background including the wet clutch system, as well as current feed forward control strategy; section 3 will propose the fine tuning method based on the genetic algorithm; section 4 will show the fine tuning results; section 5 will draw the concluding remarks. 


\section{Background}

\subsection{Wet Clutch System and Test Bench}

A wet clutch is a clutch that its friction plates are immersed in a cooling lubricating fluid which keeps the surfaces clean and gives smoother performance and longer life. Fig. 1 shows the design of a wet clutch. An electro-hydraulic pressure-regulated proportional valve regulates the pressure inside the clutch, such that the position of a piston which presses the multiple clutch disks together can be controlled. In the closing process of a wet clutch, the piston should be placed as far as possible from the clutch disks to avoid energy loss due to viscous friction of the oil between the disks. A returning spring inside of the clutch could keep the piston in this position when the clutch is not actuated. Once the command to engage the clutch is received, the left chamber of the clutch is filled with oil, so that the piston is pushing forward.

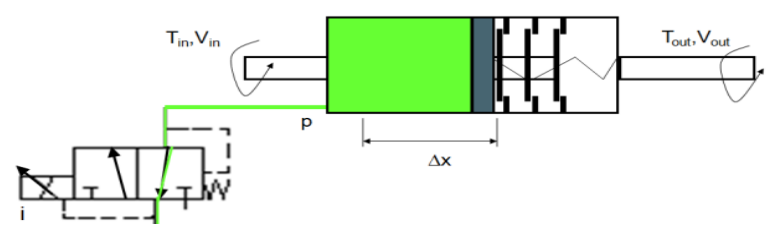

Fig. 1. The mechanical design of a wet clutch, $i$ is the triggle signal for the valve, $p$ is the flow pressure to push the piston, $T_{\text {in }}$ and $V_{\text {in }}$ are the input torque and velocity, $T_{\text {out }}$ and $V_{\text {out }}$ are the output torque and velocity, and $\Delta x$ is the displacement of the piston

When evaluating the engagement performance of a wet clutch, it is necessary to consider both fast and smooth. One typical performance of the clutch is shown in Fig. 2. The unit for the torque measurement is $N \cdot m$, while the unit for the output speed is rpm. For confidential reasons, all results in this paper will be scaled. And thus the objectives to be optimized can be defined numerically in (1) and (2).

$$
\begin{gathered}
T_{f}=\left[\text { number of samples }\left(\text { output speed }<v_{\text {out }}\right)\right] \\
\Gamma=\mid \text { min }(\text { toruqe }) \mid
\end{gathered}
$$

The test bench used in this paper contains an electromotor and a flywheel. The electromotor drives a flywheel via two mechanical transmissions: one transmission is controlled in this project; the other transmission is used to vary the load and to adjust the braking torque. The sampling frequency on this setup is $1000 \mathrm{~Hz}$. 


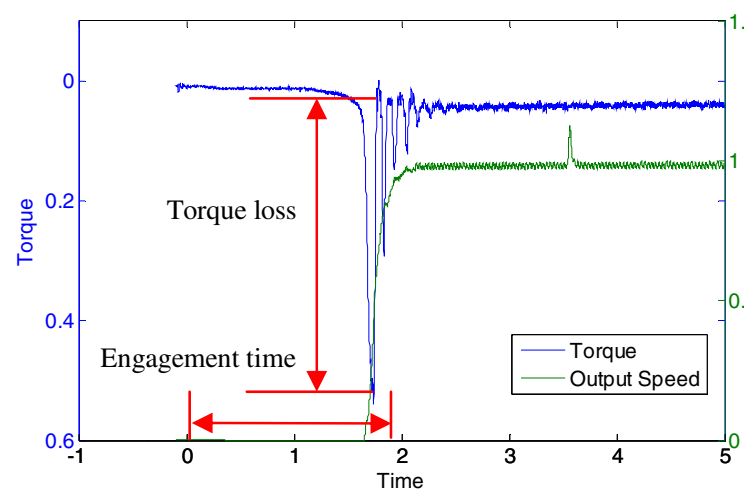

Fig. 2. Illustrative example of the performance of a wet clutch

\subsection{Current Control Strategy}

Currently, wet clutches used in industry are filled with a feed-forward parametric signal of the current to the electro-hydraulic valve. Fig. 3 shows a typical parameterized, feed-forward current signal, which is sent to the valve [1].

The shape of this signal perfectly illustrates the underlying idea behind the actual industrial control design. First, a step signal with height $a$ and width $b$ is sent to the valve to generate a high pressure level in the clutch. With this pressure, the piston will overcome the force from the return spring, and start to get closer to the clutch disks. After this pulse, the signal will give some lower current with height $c$ and width $d$ to decelerate the piston and trying to position it close to the clutch disks. Once the piston is close to the clutch disks and with very low velocity, a force is needed to push the piston forward so that the clutch disks are compressed together. This force will be generated by the pressure which is caused by the step current with height $e$ and width $f$. Then a ramp current signal with slope alpha and the end height $g$ is sent to the valve so that the pressure inside the clutch will increase again gradually. In order to secure the full closing of the clutch, the signal will be kept as a high current level afterwards. Many research efforts can be found in tuning such kind of signals in order to achieve a good engagement $[2,3]$.

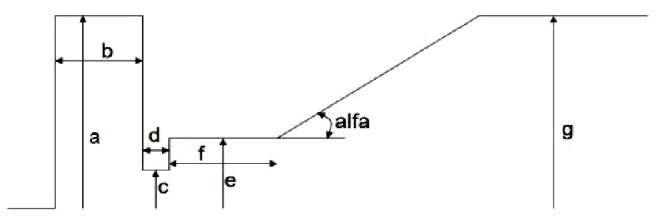

Fig. 3. Typical parameterized signal for controlling the wet clutch system 


\section{$3 \quad$ Fine Tuning the Signal}

\subsection{Brief Introduction of the Proposed Tuning Method}

In this paper, a fine tuning method mainly based on Genetic Algorithm (GA) will be proposed to solve this problem. In this new method, the values of the parameters' will be first determined by using a non-dominate sorting genetic algorithm (NSGA) [4, 5]. Then the Fast Fourier transform (FFT) will be applied on the desired signal which is selected by using a user defined weight factor, and its nearest neighbors. Within the obtained Pareto front, one focus group is selected by using a user defined weight vector. A band limited signal composed by harmonics is used to approximate these signals. By using discrete Fourier transform (DFT) [6, 7], these signals/individuals within the focus group will give the ranges of the corresponding frequencies. These amplitudes of the signals will become the tuning parameters in the fine tuning stages, while the range is also determined by the upper and lower limit get from the focus group. GA will be employed again to tune these parameters. A weighted sum method $[8,9]$ will be used here to turn the multi-objective optimization into a single objective one. After the final optimized parameters are obtained, the inverse discrete Fourier transform (IDFT) [10] is used to convert back into the signal. The final signal is expected to give a better performance.

\subsection{Forming the Pareto Front}

The genetic algorithm (GA) is a search heuristic that mimics the process of natural evolution. Recently, GA has received considerable attention as a novel approach to multi-objectives optimization problems, resulting in a fresh body of research and applications. Typically, there are two ways in GA for handling the multi-objectives optimization problems; one is use the weighted sum method to transfer the multiobjectives problem to a single objective problem; the other one is based on the nondominated sorting method to find out the Pareto front solution. The nondominated sorting Genetic Algorithm (NSGA) appears to achieve the most attention in the evolutionary algorithm literature and has been used in various studies. The key point of this method is the nondominated sorting and the fitness assignment. Before the selection is performed, the population is ranked on the basis of an individual's nondomination. Nondomination here is defined as: in a minimization problem, if a vector $X 1$ is partially less than $X 2$, we say $X 1$ dominates $X 2$, and any member of such vectors that is not dominated by any other member is said to be nondominated [4]. Then dummy fitness value is assigned according to the rank, individuals in the same rank share same fitness value. 


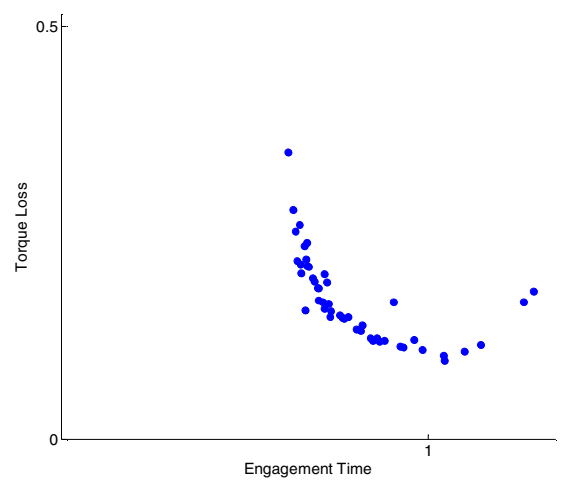

Fig. 4. Individuals of last generation

Table 1. GA parameters

\begin{tabular}{ll}
\hline Number of Decision Variables & 4 ( in Fig. 3, parameter $b, c, d$ and $f$ ) \\
Number of Individuals & 50 \\
Maximum Number of Generations & 50 \\
Number of Objectives & 2 \\
Crossover Rate & 0.9 \\
Mutation Rate & 0.1 \\
\hline
\end{tabular}

After determined the objectives for optimization (in section 2.1), the NSGA is running. The parameters in running the GA are shown in Table 1. The GA is manually stopped at generation eight since over $90 \%$ of the individual within the generation converges at the so called Pareto front (Fig. 4).

\subsection{Defining the Fine Tuning Objectives and Parameters}

Along the Pareto front, the performance of the clutch shows different characteristics. The individuals gathering at the upper left area give an aggressive engagement which will experience the fast closure of the clutch disks and a large torque loss. On the other hand, the individuals laying on the lower right part could provide a smoother engagement in the cost of time. The Pareto front can offer the flexibility of in choosing the proper signals with different requirements. When in the practical usage, the desired signal is selected by a user defined weight vector $w=\left(w_{1}, w_{2}\right), w_{1}$ and $w_{2}$ are the respected weight of two objectives and yields

$$
w_{1}+w_{2}=1
$$

The fine tuning targets are then defined as:

$$
\{P \| P-l \mid<\sigma, P \in \text { Pareto front }\}
$$

In which $\sigma$ is the distance and $l$ is the line defined as:

$$
l: y=\left(w_{1} / w_{2}\right) x
$$


In this study, we treat the objective 1 (engagement speed) and objective 2 (torque loss) with same importance. Thus, the weight vector is defined as $4 w_{1}=w_{2}=0.5$ (due to the difference scaling factors for both objectives). The distance is selected at 0.05 (scaled value). The selected focus group is shown in Fig. 5 with red color.

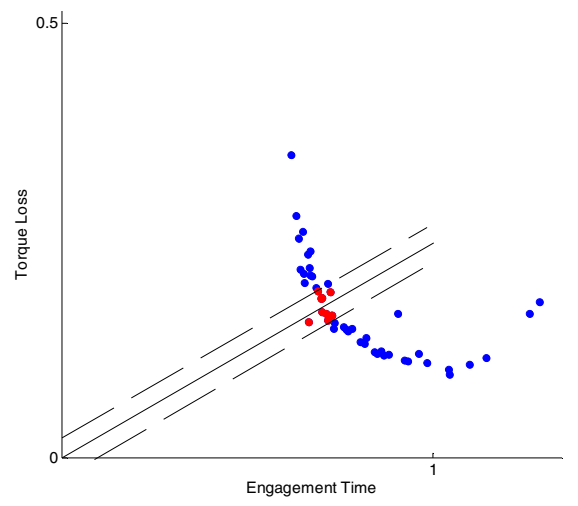

Fig. 5. Individuals as the fine tuning targets (focus group, in red)

The shape of the signals within the focus group is shown in Fig. 6. The rough shape of the signals belonging to the focus group share some similarities, the differences located on the length of first step and the height of the second step.

If we take the first second of the above signals as one period from a periodical signal, then it can be decomposed into a Fourier series [11]. This transform could bring the signal function from the time domain to the frequency domain. Using a Fourier series, the signal $x(t)$ in the time domain can be expressed as a sum of sines and cosines of different frequencies:

$$
x(t)=\sum_{-\infty}^{\infty} c_{k} e^{j 2 \pi k f t}
$$

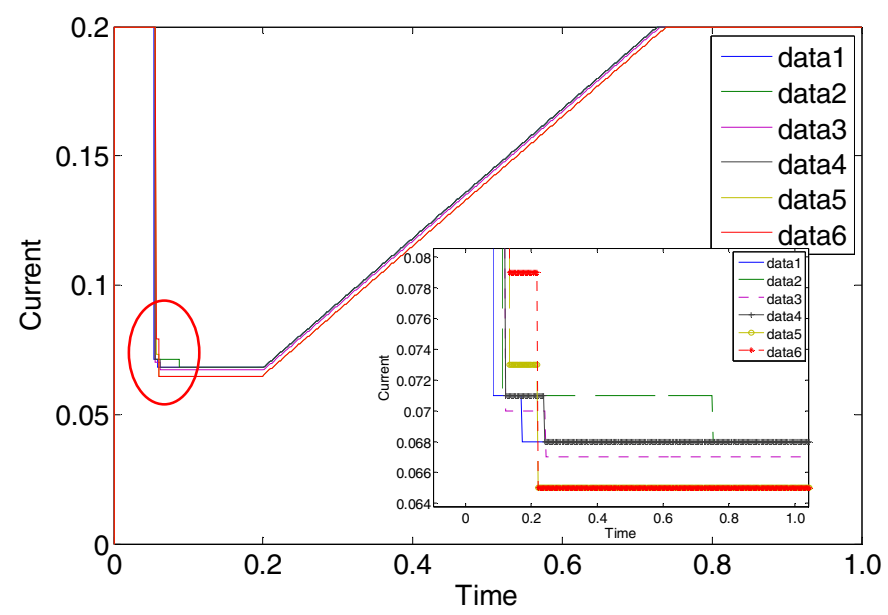

Fig. 6. Signals' shapes of focus group 


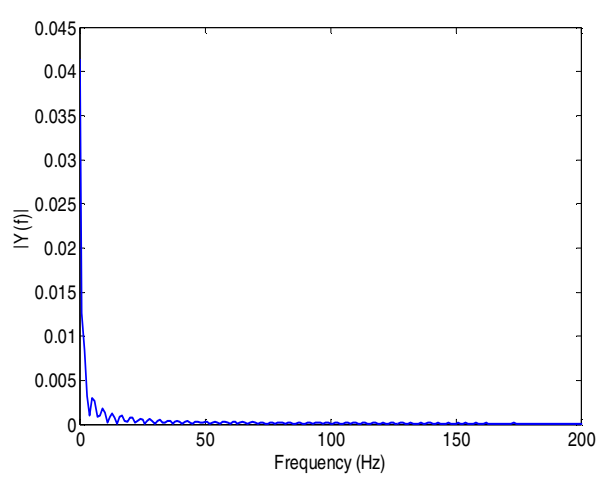

(a)

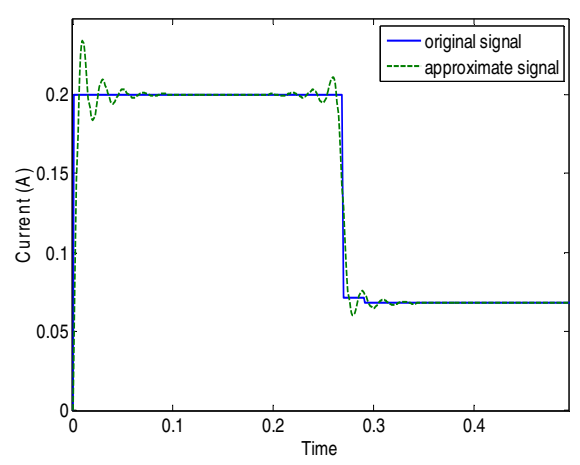

(b)

Fig. 7. (a) Spectrum analysis for target signal, single-sided; (b) Original signal and approximated signal

One way to determine the frequency range of the harmonics to form the approximated signals is using the DFT, we can analysis the individuals within the focus group in order to get the information about theirs spectrums. From the results of spectrum analysis (Fig. 7(a)), we can notice that it is not necessary to keep all the harmonics from the transform since in the high frequency range, the amplitudes are very small. Thus, instead of taking the whole frequency range, the first 50 harmonics are used to generate a band limited signal which is approximating the original signal by using the inverse discrete Fourier transform (IDFT). The original part of the signal has a length of $1 s$, which gives a fundamental of $1 / 1=1 \mathrm{~Hz}$. By taking the first 50 harmonics, the bandlimited for the approximated signal will be $50 \mathrm{~Hz}$. The approximated signal and the original signal are shown in Fig. 7(b).

In such presentation, the approximated signal has 50 harmonics; the amplitudes of these 50 harmonics offer the possibility for the fine tuning. The tunable parameters are the amplitudes and the range for every amplitude is defined as:

$$
c_{i j k} \in\left[\min \left(c_{k}\right), \max \left(c_{k}\right)\right]
$$

In which, $c_{i j k}$ is the amplitude for $k$ th harmonic of $j$ th individual in $i$ th generation. And the $c_{k}$ is the amplitude vector for $k$ th harmonics which is obtained by transforming the individuals within the focus group.

\subsection{Fine Tuning Process}

After determine the decision variables and the range of them, genetic algorithm again is used to find out the optimized combinations. Unlike the NSGA used in section 2.1, this time a weight vector will be added here so that this multi-objective problem will be transformed into a single objective problem. The new objective is defined as: 


$$
O b j=w_{1} O b j_{1}+w_{2} O b j_{2}
$$

Now the optimization becomes minimize $(O b j)$. In order to accelerate the convergence, penalty rules are added. For individual $P_{i}$, if either $O b j_{i 1}>O b j_{1 \text { max }}$ or $O b j_{i 2}>O b j_{2 \text { max }}$, the final objective value of such individual will be set very high. In which, $O b j_{j \max }$ is defined as:

$$
O b j_{\text {max }}=\left\{\max \left(O b j_{j}\right) \mid P, j=1,2\right\}
$$

The individuals within the focus group are treated as the initial population.

\section{$4 \quad$ Results}

The GA used in section 3.4 stopped at $6^{\text {th }}$ generation. The result is then compared with the performance of the central individual of the focus. Fig. 8 shown the signals, in which the blue line is the signal located in the center of the focus group; and the red line is the final fine tuned signal.

Fig. 9 showed the performance of the different signals. For the confidential requirement from the project partner, all the measurements are given in scaled measurement, thus the units are not provided in the figures. The output torques caused by the above two signals are illustrated. We can notice that with a further fine tuning, the performance could be optimized.

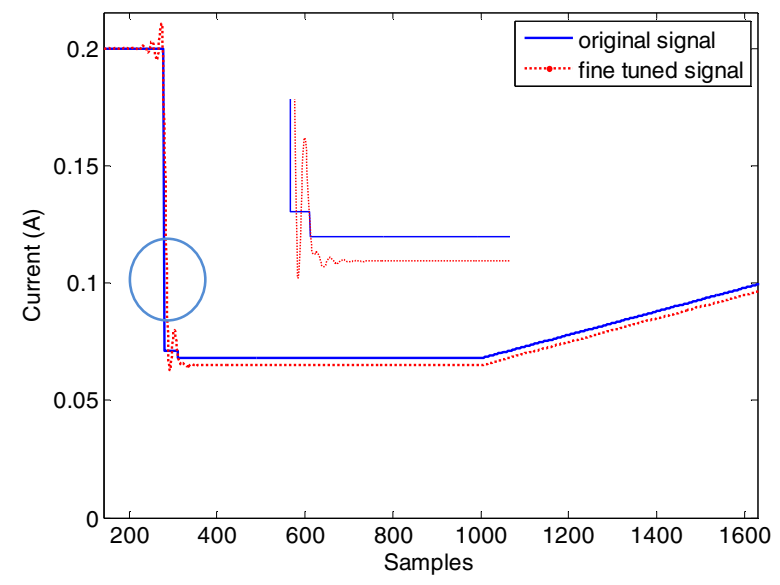

Fig. 8. The comparison of the signals 


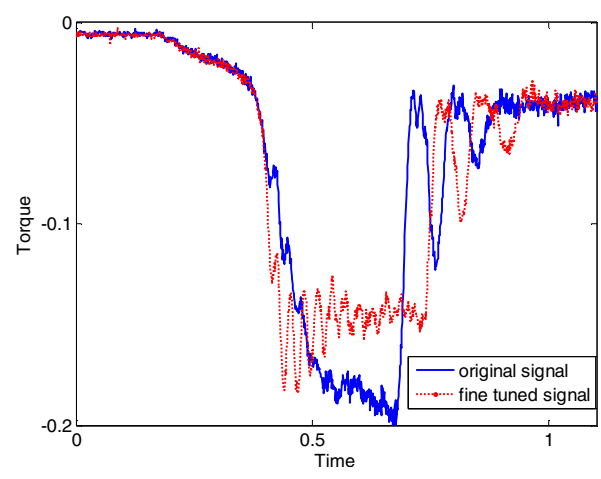

(a)

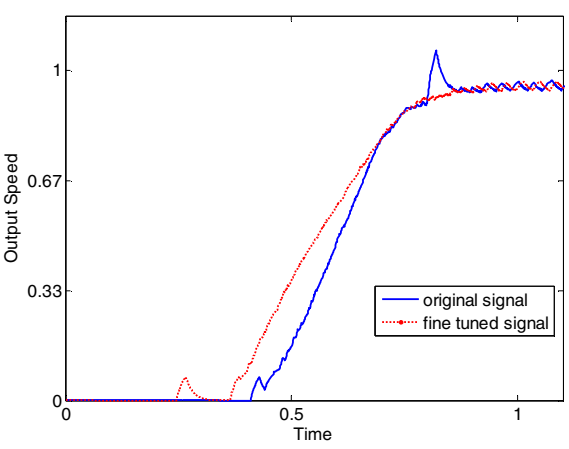

(b)

Fig. 9. (a) The comparison of the output torque; (b) The comparison of the engagement speed

\section{$5 \quad$ Concluding Remarks and Future Work}

In this paper, a new tuning method based on genetic algorithm is proposed to obtain the optimal signal profile in engaging a wet clutch system. The objective for this problem is to achieve a smooth engagement in the shortest time. Conventional, a parameterized feed forward signal which the values of those parameters are determined either empirically or through some other learning algorithms is used in this system.

This method could also be extended to optimize other feed forward parameterized signal. Besides the better performance, this tuning method can also transform the parameterized signal with sharp edges (discontinuity) into continuous signal. In some cases like the high speed motion with sudden direction changes, this property will beneficial a lot in practical usage.

Acknowledgements. This work has been carried out within the framework of the LeCoPro project (grant nr. 80032). C.M. Ionescu is financially supported by the Flanders Research Center (FWO).

\section{References}

[1] Hebbale, K.V., Kao, C.-K., McCulloch, D.E.: Adaptive electronic control of power-on upshifting in an autamatic transmission. US Patent No. 5,282,401 (1994)

[2] Depraetere, B., Pinte, G., Swevers, J.: Iterative optimization of the filling phase of wet clutches. In: The 11th International Workshop on Advanced Motion Control, Nagaoka, Japan, pp. 94-99 (2010)

[3] Lazar, C., Caruntu, C.-F., Balau, A.-E.: Modelling and predictive control of an electrohydraulic actuated wet clutch for automatic transmission. In: IEEE International Symposium on Industrial Electronics, Bari, Indonesia, pp. 256-261 (2010)

[4] Srinivas, N., Deb, K.: Multiobjective function optimization using nondominated sorting genetic algorithms. Evolutionary Computation 3, 221-248 (1995) 
[5] Fonseca, C.M., Fleming, P.J.: Multiobjective optimization and multiple constraint handling with evolutionary algorithms I: A unified formulation., Reasearch report 564, Department of Automatic Control and Systems Engineering, University of Sheffield (1995)

[6] Oppenheim, A.V., Schafer, R.W., Buck, J.R.: Discrete-time signal processing. Prentice Hall, Upper Saddle River (1999)

[7] Brigham, E.O.: The fast Fourier transform and its applications. Prentice Hall, Englewood Cliffs (1988) ISBN 0-13-307505-2

[8] Ishibuchi, H., Murata, T.: A multiobjective genetic local search algorithm and its application to flowshop scheduling. IEEE Transactions on System, Man, and Cybernetics 28(3), 392-403 (1998)

[9] Gen, M., Cheng, R.: Genetic algorithms and engineering optimization. John Wiley \& Sons, New York (2000) ISBN 0-471-31531-1

[10] Duhamel, P., Piron, B., Etcheto, J.M.: On computing the inverse DFT. IEEE Transactions on Acoust., Speech and Signal 6(2), 285-286 (1988)

[11] Proakis, J.G., Manolakis, D.G.: Digital signal processing, principles, algorithms, and applications, 3rd edn. Prentice Hall (1996) ISBN 0-13-394338-9 